\title{
Implementation and evaluation of a nurse-driven noninvasive ventilation weaning protocol in infants with severe bronchiolitis
}

\author{
Julie Cassibba ${ }^{1}$, Marie Chevallier ${ }^{2}$, ISABELLE PIN ${ }^{2}$, Aurélie Alexandre ${ }^{2}$, Alice Fumagalli², \\ Brigitte Fauroux ${ }^{3}$, and guillaume mortamet ${ }^{4}$ \\ ${ }^{1}$ Grenoble Alpes University Hospital Centre Couples and Children Section \\ ${ }^{2}$ Centre Hospitalier Universitaire Grenoble Alpes Pôle Couple Enfant \\ ${ }^{3}$ Hopital universitaire Necker-Enfants malades \\ ${ }^{4}$ Centre Hospitalier Universitaire Grenoble Alpes
}

January 8, 2021

\begin{abstract}
Rationale: Noninvasive ventilation (NIV) is the first-line therapy in infants with bronchiolitis-related acute respiratory failure. However, there is a lack of data regarding weaning from NIV in this setting. Working hypothesis: This study aims to evaluate a nurse-driven weaning protocol in this homogenous population. Study design: A retrospective single-center study with preversus-post comparative design in a tertiary center. Methodology: Data from all infants aged [?] 6 months admitted to the PICU during 2 seasons with a clinical diagnosis of bronchiolitis and requiring any type of noninvasive ventilatory support on admission, were analyzed. Main results: In total, 187 infants (95 with standard and 92 with nurse-driven protocols) were included; the median age was 47 (IQR 24-75) and 31 days (19-58) in patients at baseline and after the protocol implementation, respectively. There was no difference in terms of weaning failure between the two periods (11 (12\%) versus $14(15 \%)$, $\mathrm{p}=0.46)$. At baseline, the ventilatory support duration was 70 hours (IQR 54-104) versus 56 hours (IQR 29-83) during the nurse-driven protocol period $(\mathrm{p}=0.29)$. The PICU and hospital lengths of stay did not differ between the two periods. No complication related to NIV occurred in the two periods. Conclusions: In patients with bronchiolitis supported by NIV, the nurse-driven weaning management - as opposed to physician-driven - was not associated with a significantly higher proportion of weaning failure cases.

Title: Implementation and evaluation of a nurse-driven noninvasive ventilation weaning protocol in infants with severe bronchiolitis

Authors: Julie Cassibba ${ }^{1}$, Marie Chevallier ${ }^{2}, \mathrm{MD}, \mathrm{PhD}$, Isabelle $\mathrm{Pin}^{1}$, MD, Aurélie Alexandre ${ }^{1}$, Alice Fumagalli $^{1}$, RN, Brigitte Fauroux ${ }^{3,4}, \mathrm{MD}, \mathrm{PhD}$, Guillaume Mortamet ${ }^{5,6}$, MD, PhD

${ }^{1}$ Pediatric Department, Grenoble Alpes University Hospital, Grenoble, France

${ }^{2}$ Neonatalogy Depratement, Grenoble Alpes University Hospital, Grenoble, France

${ }^{3}$ Pediatric noninvasive ventilation and sleep unit, AP-HP, Hôpital Necker-Enfants Malades, Paris

${ }^{4}$ Université de Paris, VIFASOM, Paris, France

${ }^{5}$ Inserm U1042 unit, Grenoble Alpes University, Grenoble, France

${ }^{6}$ Pediatric Intensive Care Unit, Grenoble Alpes University Hospital, Grenoble, France
\end{abstract}

\section{Corresponding author:}

Dr Guillaume Mortamet 
Pediatric Intensive Care Unit, CHU Grenoble Alpes, 38700 La Tronche, France

E-mail: gmortamet@chu-grenoble.fr

Telephone: +33476765503

Funding:

None

Meeting

This has never been presented to a meeting

Keywords: Infants, Noninvasive Ventilation, Acute respiratory Failure, Nurse-driven, Weaning, Pediatric Intensive Care Units

\section{Running title}

Noninvasive ventilation weaning protocol

\section{Competing interest}

JC, MC, IP, AA, AF, BF and GM declare that they have no conflict of interest.

\section{Authors' contribution:}

AA, AF and GM built the protocol. JC and GM collected the data. JC, MC, IP and BF wrote the manuscript, which was reviewed, edited, and approved by all the authors.

As the corresponding author, GM has final responsibility for the decision to submit for publication.

Words count of manuscript: 2385

Words count of abstract: 220

\section{Abstract:}

Rationale: Noninvasive ventilation (NIV) is the first-line therapy in infants with bronchiolitis-related acute respiratory failure. However, there is a lack of data regarding weaning from NIV in this setting.

Working hypothesis: This study aims to evaluate a nurse-driven weaning protocol in this homogenous population.

Study design: A retrospective single-center study with pre-versus-post comparative design in a tertiary center.

Methodology: Data from all infants aged [?] 6 months admitted to the PICU during 2 seasons with a clinical diagnosis of bronchiolitis and requiring any type of noninvasive ventilatory support on admission, were analyzed.

Main results: In total, 187 infants (95 with standard and 92 with nurse-driven protocols) were included; the median age was 47 (IQR 24-75) and 31 days (19-58) in patients at baseline and after the protocol implementation, respectively. There was no difference in terms of weaning failure between the two periods (11 $(12 \%)$ versus $14(15 \%), \mathrm{p}=0.46)$. At baseline, the ventilatory support duration was 70 hours (IQR 54104) versus 56 hours (IQR 29-83) during the nurse-driven protocol period $(\mathrm{p}=0.29)$. The PICU and hospital lengths of stay did not differ between the two periods. No complication related to NIV occurred in the two periods.

Conclusions: In patients with bronchiolitis supported by NIV, the nurse-driven weaning management - as opposed to physician-driven - was not associated with a significantly higher proportion of weaning failure cases.

\section{Introduction}


Acute bronchiolitis is a severe infection involving young children and a leading cause of hospitalization worldwide ${ }^{1,2}$. Since management of severe bronchiolitis is mainly based on mechanical ventilation, most of patients with bronchiolitis-related acute respiratory failure require admission in a Pediatric Intensive Care Unit (PICU) ${ }^{3}$. Noninvasive Ventilation (NIV), including both Continuous Positive Airway Pressure (CPAP) and Bilevel Positive Airway Pressure (BiPAP), is commonly used in infants with severe bronchiolitis 3,4. Alternatively, High-Flow Nasal Cannula (HFNC) is increasingly used in infants with more moderate respiratory failure ${ }^{5,6}$.

Despite the large use of NIV in infants with bronchiolitis, there is a paucity of data regarding weaning procedures and weaning initiation criteria in this population ${ }^{7}$. However, these aspects of ventilatory support are of crucial importance since maintaining a non-necessary ventilatory support may be associated with prolonged hospitalization in PICU and greater health care costs. Nevertheless, premature weaning may increase the morbidity and the duration of ventilation ${ }^{8}$. Thus, there need to be a balance between ensuring that NIV is not unnecessarily prolonged and protecting infants from weaning failure.

It has been shown that protocolized weaning from invasive mechanical ventilation may be associated with a reduction in duration of mechanical ventilation ${ }^{9}$. However, no study has been performed in children supported by any type of NIV. PICU nurses spend more time at the bedside of patients as compared to doctors, especially during night-shifts when physicians are responsible for more patients. In facts, effective weaning requires a close collaboration between nurse and physician to screen the patient, chose the appropriate moment and perform en adequate monitoring of the patient after weaning. Despite a large variability between European countries, Tume et al.reported that nurses have a key role and an influence on ventilation and weaning decisions in critically ill children ${ }^{10}$.

We hypothesized that input of PICU nurses might be valuable for the initiation and follow-up of NIV weaning in infants with acute viral bronchiolitis supported by any type of noninvasive ventilatory support. In this context, the primary objective of the study was to implement a nurse-driven NIV weaning protocol and to evaluate its feasibility as compared to a standard doctor-driven protocol.

\section{Methods}

\section{Study design}

This single-center retrospective observational study with pre-versus-post comparative design was conducted over two bronchiolitis seasons, from November 2018 to April 2020, in a 16-beds PICU of a tertiary pediatric center. The nurse to children ratio was $1: 2$ or 1:3, 24/7, during this period.

The study compared two bronchiolitis seasons: a first season (2018-2019) during which the infants were weaned according to the routine (doctor-driven) practice of the unit, and a second season (2019-2020) during which the infants were weaned according to the nurse-driven protocol.

The study was approved by the regional research committee (Comite d'Ethique du Centre d'Investigation Clinique de Clermont-Ferrand, France, IRB 5891) and follows the STROBE guidelines.

\section{Study population}

The medical charts of all infants aged [?] 6 months admitted to the PICU during the 2 seasons with a clinical diagnosis of bronchiolitis and requiring any type of ventilatory support, including CPAP, BiPAP or HFNC (with an air flow rate $>1 \mathrm{~L} / \mathrm{kg} / \mathrm{min}$ ), on admission, were analyzed. The admission criteria in PICU were left at the discretion of the attending physician without any local protocol. Patients were excluded if they were on long-term CPAP or BiPAP ventilation, if they presented a cardiac or neuromuscular disorder, or a pneumothorax on the chest radiography performed at admission.

In practice, a step-up ventilator strategy was used according to the increase in patient severity, led by physicians: 1/ HFNC, 2/ CPAP, 3/ BiPAP and 4/ invasive ventilation. The local protocol for ventilatory management is detailed in Supplementary Material. CPAP and BiPAP were performed using an ICU ventilator (Evita XL, Drager(r), Germany or Servo I and U, Maquet(r), Solna, Sweden) with a facial or nasal 
interface. HFNC was started at a flow rate of $2 \mathrm{~L} / \mathrm{kg} / \mathrm{min}$ with an ICU ventilator (Evita XL, Draeger(r), Germany), or with a specific device (Airvo 2, Fisher and Paykel(r), Auckland, New Zealand). In our center, HFNC is not used in pediatric wards. Patients did not receive any sedative drug and continuous nasogastric tube feeding was started at least 6 hours after admission. The parents are allowed and encouraged to stay with their child $24 / 7$ in order to reassure their infant.

Demographic (age, sex and comorbidities) and clinical data during the PICU stay were collected. Respiratory comorbidity was defined as any clinically significant neonatal lung disease, such as pulmonary bronchodysplasia. Clinical data at admission were the Pediatric RISk of Mortality score (PRISM), the Wang ${ }^{11}$ and the m-WCAS (modified Wood's Clinical Asthma Score) bronchiolitis clinical severity scores ${ }^{12}$, hemodynamic and respiratory parameters, ventilatory mode and settings and medical treatment. Biological data on admission, including venous $\mathrm{pH}$, venous carbon dioxide $\left(\mathrm{PvCO}_{2}\right)$ and ventilatory settings at admission were also gathered. All infants were screened for respiratory syncytial virus (RSV) at admission. The rate of weaning failure, the rate of intubation, the duration of ventilator support, the duration of the weaning process, a suspicion of bacterial co-infection, defined as the presence of new or changing infiltrates, fever associated with a significant increase in biological inflammatory markers, and the PICU and hospital lengths of stay were analyzed.

\section{Doctor-driven protocol (first year)}

During the first season (November 2018 to April 2019), the weaning from ventilatory support followed the usual doctor-driven protocol and was based on a step-down strategy from BiPAP to CPAP, with the possibility to use HFNC as an intermediate mode of support. The decision to start weaning was based on the infant's clinical status. Weaning was initiated in a fully awake infant, thus after an adequate sleep period, and preferentially during the day. During the weaning period, the infant was evaluated by the doctor at least every 6 hours, and by the nurse at least every 4 hours. Nurses could suggest to the attending physician a change in the type and settings of ventilatory support but they were only allowed to modify the $\mathrm{FiO}_{2}$ on their own initiative.

\section{Nurse-driven protocol (second year)}

The nurse-driven protocol was implemented during the second season (November 2019 to April 2020). The protocol has been set up by a team comprising a senior pediatric intensivist (GM), a PICU nurse (AF) and a physiotherapist (AA) and was inspired from a recently published survey ${ }^{7}$. The final protocol was approved by all PICU nurses, residents, fellow and senior physicians before its implementation.

The protocol followed also a step-down strategy with an obligatory switch from BiPAP to CPAP or HFNC according to the physician in charge based on the infant's clinical status, for at least 12 hours, and before being included in the nurse-driven protocol. A support-free trial was only allowed once the patient was on CPAP or HFNC. The protocol could be carried out at least 12 hours after PICU admission and included a five-step algorithm (Figure 1):

1/the PICU nurse assessed whether the child fulfilled or not the eligibility criteria to start the weaning protocol

$2 /$ she/he asked for the doctor's agreement

3 / she/he assessed the presence of all weaning criteria $\left(\mathrm{SpO}_{2}\right.$ [?] $94 \%$ with $\mathrm{FiO}_{2}$ [?] $35 \%$, respiratory rate at rest [?] 60/min, Wang score at rest [?] 7, no apnea or bradycardia or discomfort, awake and alert and normal hemodynamic status, as defined as a normal heart rate, blood pressure and capillary refill time)

4/ weaning of the support ventilatory support was carried out and

5/ the nurse assessed hourly the presence of one of the following criteria associated with NIV failure: apneas, bradycardia, sweating, increase in blood pressure, oxygen $>1 \mathrm{~L} / \mathrm{min}$ for a $\mathrm{SpO}_{2}>94 \%$, an increase of Wang score of more than 2 points, an increase of respiratory rate of more than $20 \mathrm{breath} / \mathrm{min}$ ). 
In case of weaning failure, the nurse restored the previous ventilatory support and informed the attending physician. Eligibility for weaning was then reassessed 6 hours later. In case of weaning success, the infant was left without any ventilatory support; low-flow oxygen could be used to maintain a $\mathrm{SpO}_{2}>94 \%$. Weaning could be initiated during the day or night, and preferentially after an adequate sleep period.

\section{Study Outcomes}

The primary outcome was the rate of weaning failure at the first attempt. In the physician-driven group,

the rate of weaning failure within 48 hours after the weaning initiation was defined by the need to switch back to the previous ventilatory support whatever it was (CPAP or HFNC) or need to intubate the patient. The secondary outcomes were:

1/the duration of NIV (recorded to the hour) defined as the time elapsed between the onset of any type of ventilatory support (CPAP or BiPAP or HFNC) and the complete weaning from any type of ventilator support (i.e. switch in room air or with low-flow oxygen)

$2 /$ the duration of the weaning process (recorded to the hour) defined as the time elapsed between the onset of the first decrease in ventilatory mode or parameters to the complete weaning from any type of ventilator support

3/the PICU and hospital lengths of stay (recorded to the hour for PICU length of stay and to the day for hospital length of stay)

4/the suspicion of bacterial co-infection.

\section{Statistical analysis}

The data of the two cohorts, doctor-driven and nurse-driven, were compared. Descriptive data were presented as number/percentages for categorical data and median values (with interquartile range, IQR) for continuous data. Differences in categorical variables were tested using the Chi-square or Fisher's exact test. Differences in continuous variables were assessed by Student's t test, Mann-Whitney test or analysis of variance test after verification of the eligibility conditions. Difference in proportions with confidence interval was calculated using https://www.statology.org/confidence-interval-difference-in-proportions-calculator/. P values of less than .05 were considered significant. All statistical analyses were performed using SPSS 26.0 (SPSS, Inc, Chicago, IL).

\section{Results}

\section{Characteristics of the patients}

The data of 95 and 92 infants weaned with the doctor-driven protocol and the nurse-driven protocol, respectively, were analyzed (Table 1). No patient was excluded. During the second period, the nurse-driven protocol was followed in all the patients. The characteristics of the patients were comparable between the two groups, except for the age, weight and mode of respiratory support at admission. Indeed, the infants of the nurse-driven protocol were significantly younger (31 (19-58) versus 47 (24-75) days, $\mathrm{p}=0.04)$, with a lower weight $(4.1(3.5-4.8)$ versus $4.3(3.7-5.2, \mathrm{p}=0.02)$, and less use of $\operatorname{BiPAP}(\mathrm{n}=28(30 \%)$ versus $n=46$ $(48 \%), \mathrm{p}=0.04)$ as compared to the infants of the doctor-driven protocol. Ventilatory settings at ventilatory support initiation were not significantly different (Table 1).

\section{Primary and secondary outcomes}

As shown in Table 2, we found that 11/95 (12\%) of cases managed by physicians failed weaning versus 14/92 (15\%) of cases managed by nurses. The nurse-driven weaning management - as opposed to physician-driven - was not associated with a significantly higher proportion of weaning failure cases since the difference was $3 \%$ (95\% confidence interval $-0.07 / 0.13, \mathrm{p}=0.46)$. As well, the duration of the weaning process and the total duration of any type of ventilator support, as well as the PICU and hospital length of stay, were comparable between the two groups (Table 2). No patient was intubated and no complication related to NIV occurred 
in any patient. The only difference was a lower rate of suspected bacterial co-infection in the infants of the nurse-driven protocol, with a $\mathrm{p}$ at the limit of significance $(\mathrm{n}=24(27 \%)$ versus $\mathrm{n}=37(39 \%), \mathrm{p}=0.05)$, as compared to the infants of the doctor-driven protocol.

\section{Discussion}

Weaning from NIV is of major importance in the management of infants with acute respiratory failure related to bronchiolitis ${ }^{3}$. The present study with pre-versus-post design shows that the implementation of a nurse-driven weaning protocol for infants with bronchiolitis was not associated with a significantly higher proportion of weaning failure cases as compared to standard practice, i.e. a doctor-driven protocol.

Since infants with bronchiolitis represent a relatively homogeneous population with a generally favorable outcome ${ }^{3}$, we hypothesized that PICU nurses could play a key-role in the respiratory management. They spent more time at the infant's bedside; they see how the infant behave during care, with and without the ventilator support. Since the nurse-to-patient ratio is usually similar during daytime and nighttime in most PICU, as compared to physicians who are fewer during nighttime, we were interested to evaluate a greater involvement of nurses in the weaning process of these infants. Using a nurse-driven protocol as a task delegation, nurses have the possibility to decide the weaning initiation of a patient when they consider that the patient fulfills the weaning criteria, at any moment of the day and night. This procedure has several advantages. It increases the involvement and responsibility of the nurse within a strict frame (i.e. , the written protocol), its saves time for physicians, especially during periods with increased activity (night), and it may contribute to discharge the patients earlier to the ward after a successful weaning because the weaning process may be started at night. Indeed, a rapid and safe weaning from NIV is crucial. As reported by Baudin et al. , NIV and HFNC may be associated with complications, including pneumothorax, skin lesions and gastric distension ${ }^{13}$. Then, through a reduction in the duration of the weaning process, reducing the duration of ventilator support contributes to discharge patients from PICU earlier. It is therefore important to wean the infant from any type of ventilatory support as soon as possible. In the present study, the implementation of a nurse-driven protocol did not allow to reduce the absolute duration of ventilation. However, we did not analyze the moment of PICU discharge. Indeed, a successful weaning during the night may allow a discharge early in the morning which may free a bed for another patient.

To our knowledge, very few studies have analyzed NIV weaning in children. Most of them have been conducted in specific populations such as neonates or patients with long-term NIV ${ }^{14,15}$. However, patients with bronchiolitis are very different in terms of age, lung development and respiratory disease. Recently, our team conducted a survey in PICU physicians that revealed the extreme heterogeneity of NIV weaning practices in infants with bronchiolitis ${ }^{7}$. As standard practice, we use in our unit a step-down strategy using in some patients HFNC as an intermediate mode of support, which represents a high-cost strategy. In the nurse-driven protocol, weaning from CPAP was more rapid without the use of HFNC which was not associated with a higher rate of weaning failure.

In the present study, we chose weaning failure as the primary outcome since it seemed to be clinically relevant. Indeed, weaning failure may largely increase the duration of ventilation and ICU lenght of stay since the second weaning attempt is usually performed at least 6-12 hours later. In addition, the use of ICU length of stay as secondary outcome may be controversial since discharge from ICU does not only depends on patient's status, but also on beds availability in wards.

This study presents some limitations that should be highlighted. First, it is a single center study that compared two cohorts on two consecutive years, and not a controlled trial. As a retrospective study, we could not assess nor the safety neither the non-inferiority of the protocol. Indeed, we found that management of weaning by nurses - as opposed to physicians - was not associated with a significantly higher proportion of weaning failure cases but the failure to identify such a difference may be related to sample size, confounding factors and lack of adequate matching. As a retrospective study, there was no assurance that the respiratory support protocol was in facts prescriptive or followed in every patient.

Moreover, we cannot guarantee that physicians were not continuing to give input in the nurse-led epoch. 
As well, in the physician-led epoch, we cannot guarantee that nurses were active participants and could suggest to the attending physician in charge a change in the type and settings of ventilatory support. As a single-center study, the larger use of a nurse-driven weaning protocol warrants a validation in other PICU, including non-university hospitals. In addition, the infants of the nurse-driven protocol were younger and less often treated with BiPAP. A young age is associated with an increased severity and may explain the greater number of infants treated with BiPAP in the nurse-driven protocol. Despite this bias, which could disadvantage the nurse-driven group, the patients' respiratory outcome was comparable between the two groups. The moment of PICU discharge (morning, afternoon or evening) was not analyzed and neither the satisfaction of the nurses and the doctors. These two elements may however be important for the organization and teamwork in a PICU. Finally, we did not objectively measure the additional workload performed by the nurses during the nurse-driven protocol as compared to the physician-driven protocol.

In conclusion, in patients with bronchiolitis supported by NIV, the nurse-driven weaning management - as opposed to physician-driven - was not associated with a significantly higher proportion of weaning failure cases and higher NIV duration. This change in respiratory management seems worth to be evaluated in other types of respiratory failure in children.

\section{Figure legends}

Figure 1: Nurse-driven weaning protocol used in our unit. CPAP: Continuous positive airway ventilation; HFNC: High-Flow Nasal Cannula; PICU: Pediatric intensive care unit

\section{Bibliography}

1. Green CA, Yeates D, Goldacre A, Sande C, Parslow RC, McShane P, Pollard AJ, Goldacre MJ. Admission to hospital for bronchiolitis in England: trends over five decades, geographical variation and association with perinatal characteristics and subsequent asthma. Arch Dis Child 2016;101(2):140-146.

2. Fauroux B, Hascoet JM, Jarreau PH, Magny JF, Roze JC, Saliba E, Schwarzinger M. Risk factors for bronchiolitis hospitalization in infants: A French nationwide retrospective cohort study over four consecutive seasons (2009-2013). PLoS One 2020;15(3):e0229766.

3. Clayton JA, McKee B, Slain KN, Rotta AT, Shein SL. Outcomes of Children With Bronchiolitis Treated With High-Flow Nasal Cannula or Noninvasive Positive Pressure Ventilation. Pediatr Crit Care Med 2019;20(2):128-135.

4. Abboud PA, Roth PJ, Skiles CL, Stolfi A, Rowin ME. Predictors of failure in infants with viral bronchiolitis treated with high-flow, high-humidity nasal cannula therapy*. Pediatr Crit Care Med 2012;13(6):e343-349.

5. Milesi C, Essouri S, Pouyau R, Liet JM, Afanetti M, Portefaix A, Baleine J, Durand S, Combes C, Douillard A, Cambonie G. High flow nasal cannula (HFNC) versus nasal continuous positive airway pressure (nCPAP) for the initial respiratory management of acute viral bronchiolitis in young infants: a multicenter randomized controlled trial (TRAMONTANE study). Intensive Care Med 2017;43(2):209-216.

6. Hough JL, Pham TM, Schibler A. Physiologic effect of high-flow nasal cannula in infants with bronchiolitis. Pediatr Crit Care Med 2014;15(5):e214-219.

7. Suzanne M, Amaddeo A, Pin I, Milesi C, Mortamet G. Weaning from Non-Invasive Ventilation and High Flow Nasal Cannula in bronchiolitis: a survey of practice. Pediatr Pulmonol 2020.

8. Amatya S, Rastogi D, Bhutada A, Rastogi S. Weaning of nasal CPAP in preterm infants: who, when and how? a systematic review of the literature. World J Pediatr 2015;11(1):7-13.

9. Foronda FK, Troster EJ, Farias JA, Barbas CS, Ferraro AA, Faria LS, Bousso A, Panico FF, Delgado $\mathrm{AF}$. The impact of daily evaluation and spontaneous breathing test on the duration of pediatric mechanical ventilation: a randomized controlled trial. Crit Care Med 2011;39(11):2526-2533. 
10. Tume LN, Kneyber MC, Blackwood B, Rose L. Mechanical Ventilation, Weaning Practices, and Decision Making in European PICUs. Pediatr Crit Care Med 2017;18(4):e182-e188.

11. Wang EE, Milner RA, Navas L, Maj H. Observer agreement for respiratory signs and oximetry in infants hospitalized with lower respiratory infections. Am Rev Respir Dis 1992;145(1):106-109.

12. Wood DW, Downes JJ, Lecks HI. A clinical scoring system for the diagnosis of respiratory failure. Preliminary report on childhood status asthmaticus. Am J Dis Child 1972;123(3):227-228.

13. Baudin F, Gagnon S, Crulli B, Proulx F, Jouvet P, Emeriaud G. Modalities and Complications Associated With the Use of High-Flow Nasal Cannula: Experience in a Pediatric ICU. Respir Care 2016;61(10):13051310 .

14. Soonsawad S, Tongsawang N, Nuntnarumit P. Heated Humidified High-Flow Nasal Cannula for Weaning from Continuous Positive Airway Pressure in Preterm Infants: A Randomized Controlled Trial. Neonatology 2016;110(3):204-209.

15. Mastouri M, Amaddeo A, Griffon L, Frapin A, Touil S, Ramirez A, Khirani S, Fauroux B. Weaning from long term continuous positive airway pressure or noninvasive ventilation in children. Pediatr Pulmonol 2017;52(10):1349-1354.

\section{Hosted file}

Table 1 SeventPro V7 R1 clean.pdf available at https://authorea.com/users/388245/articles/ 503070-implementation-and-evaluation-of-a-nurse-driven-noninvasive-ventilation-weaningprotocol-in-infants-with-severe-bronchiolitis

\section{Hosted file}

Table 2 SeventPro article V7 R1 clean.pdf available at https://authorea.com/users/388245/ articles/503070-implementation-and-evaluation-of-a-nurse-driven-noninvasive-ventilationweaning-protocol-in-infants-with-severe-bronchiolitis 


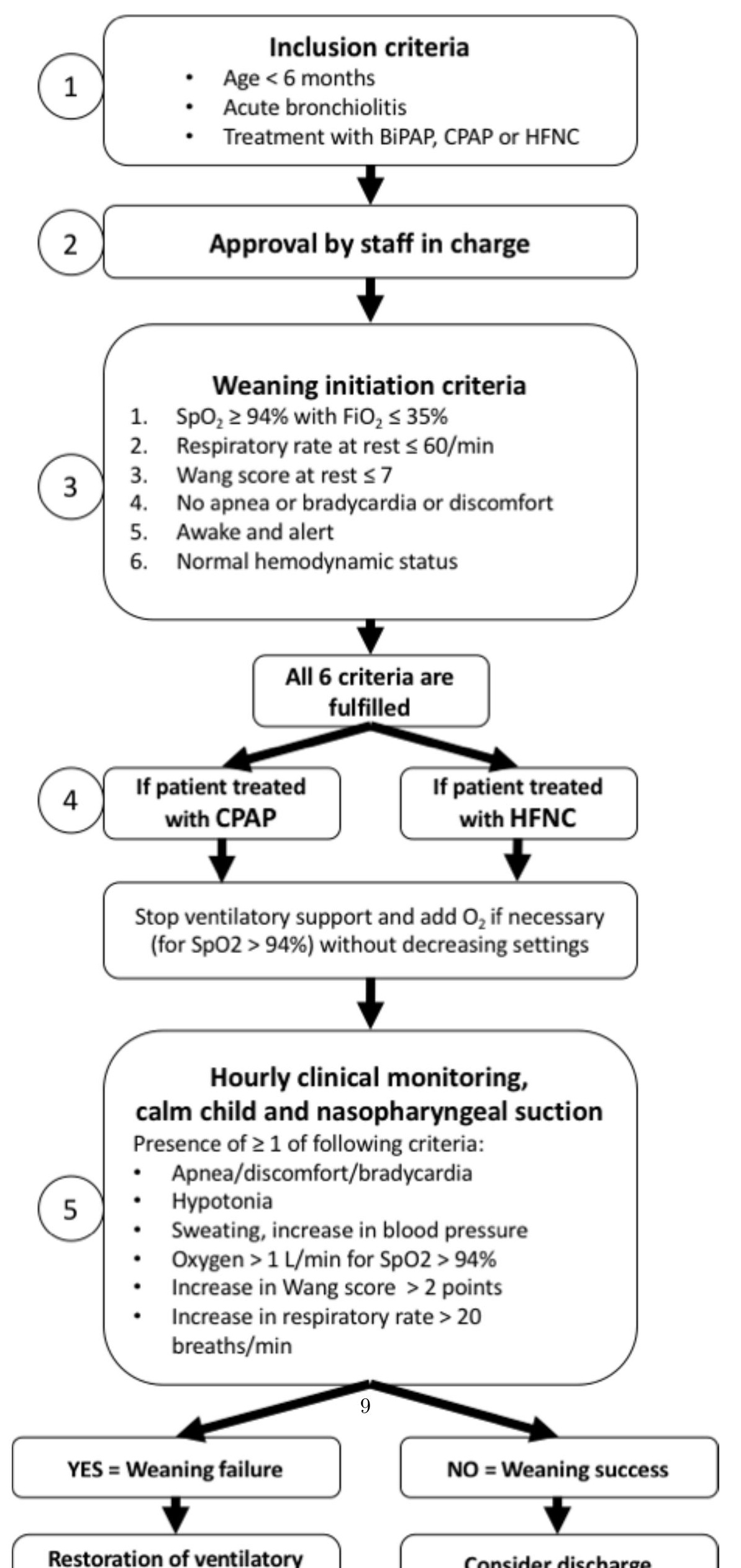

